\section{Cahiers d'ethnomusicologie}

Anciennement Cahiers de musiques traditionnelles

$18 \mid 2005$

Entre femmes

\title{
Artistes ou concubines? La tradition vocale féminine en Inde du Nord
}

Francesca Cassio

Traducteur : Ramèche Goharian

\section{OpenEdition}

Journals

Édition électronique

URL : http://journals.openedition.org/ethnomusicologie/280

ISSN : 2235-7688

Éditeur

ADEM - Ateliers d'ethnomusicologie

Édition imprimée

Date de publication : 31 décembre 2005

Pagination : $79-101$

ISSN : 1662-372X

\section{Référence électronique}

Francesca Cassio, «Artistes ou concubines ? La tradition vocale féminine en Inde du Nord », Cahiers d'ethnomusicologie [En ligne], 18 | 2005, mis en ligne le 14 janvier 2012, consulté le 19 avril 2019. URL: http://journals.openedition.org/ethnomusicologie/280

Ce document a été généré automatiquement le 19 avril 2019

Tous droits réservés 


\title{
Artistes ou concubines? La tradition vocale féminine en Inde du Nord
}

\author{
Francesca Cassio
}

Traduction : Ramèche Goharian

1 La recherche consacrée aux chanteuses de la musique hindoustanie ${ }^{1}$ est le prolongement naturel de ma précédente étude sur le dhrupad (Cassio 2000), un genre vocal de la musique classique développé en Inde du Nord du XV ${ }^{e}$ au XIX ${ }^{e}$ siècles, dont l'interprétation était dévolue à une catégorie spécifique de musiciens hommes, les voix de femmes étant jugées impropres à son exécution.

2 Base de la musique classique, le dhrupad occupe une place très importante dans la musicologie indienne. L'exclusion des femmes de ce répertoire a eu pour conséquence de marginaliser les musiciennes, depuis la tradition classique jusqu'à l'époque moderne. Une première analyse des sources orales et écrites montre effectivement que jusqu'au $\mathrm{XX}^{\mathrm{e}}$ siècle :

- le nom d'aucune femme n'est cité dans les généalogies des gharānā (dynasties de musiciens classiques où la connaissance se transmet par lignée patrilinéaire et masculine) ;

- on ne trouve pas d'enseignantes de musique et de chant comparables aux grands maîtres appelés guru ou ustad ;

- le nom d'aucune artiste ayant transmis un héritage musical n'est mentionné2. 
Fig. 1 : Un roi diverti par des musiciennes et des danseuses.

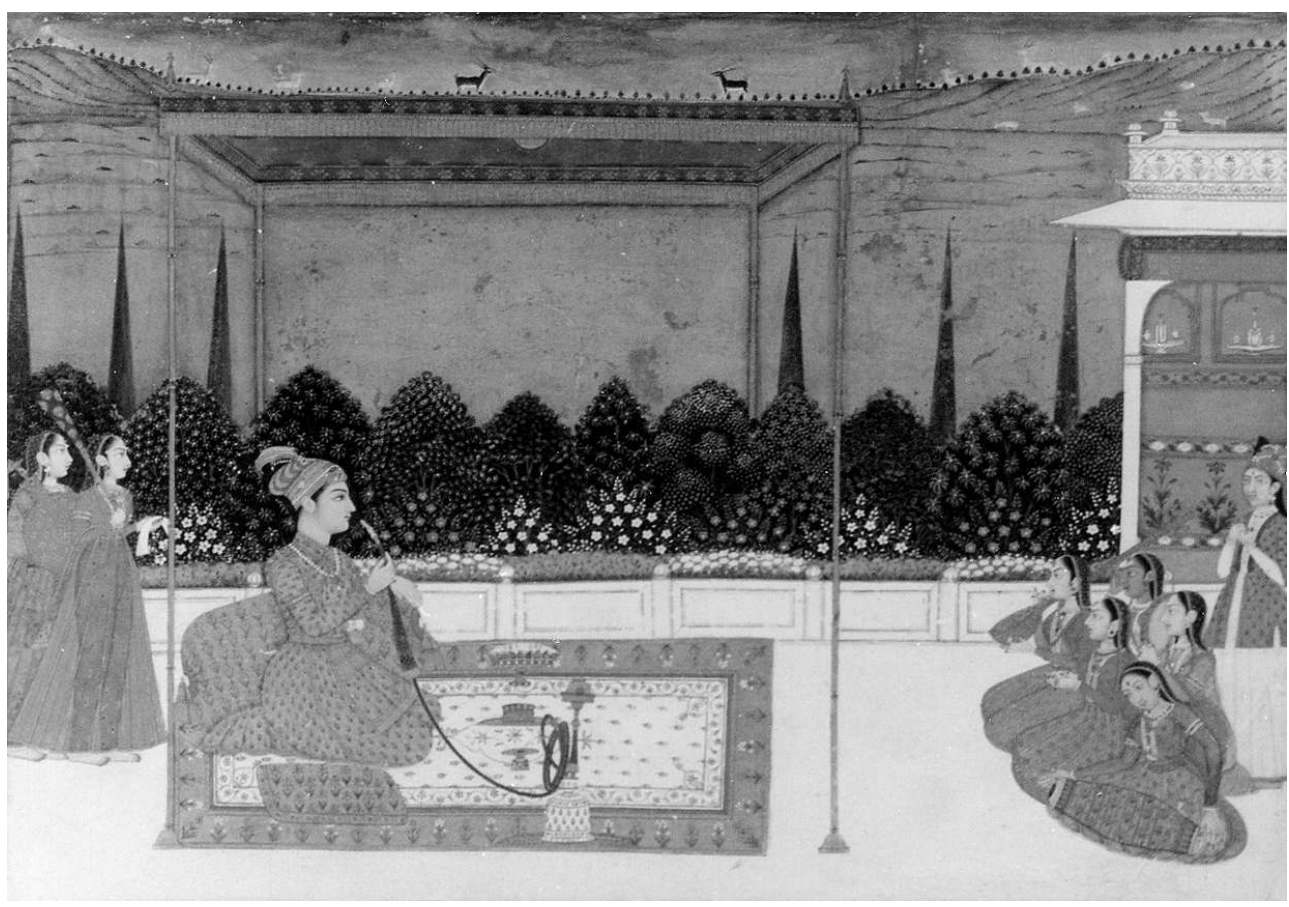

Fin du XVIII siècle (avec l'aimable autorisation de Bharat Kala Bhavan, Benares).

3 Si les femmes faisaient autrefois partie du paysage musical, leur rôle n'était toutefois pas jugé assez important pour mériter d'être mentionné, étudié ou retenu comme dans la tradition classique masculine ${ }^{3}$.

La présence de musiciennes est largement attestée dans tout le sous-continent indien par des sources iconographiques anciennes; de nombreux bas-reliefs et miniatures montrent des personnages féminins en train de jouer de la musique et danser dans des temples ou des palais. Certaines illustrations mettent en scène des spectacles privés, donnés dans l'intimité de la cour ou du gynécée; elles présentent un intérêt tout particulier car elles témoignent de la culture classique des cours princières.

En observant ces images, je me suis souvent demandé quel genre de musique était joué à ces occasions. Car même si les sources iconographiques ne nous renseignent pas sur les sons, elles attestent néanmoins l'existence d'une tradition spécifique de musique de cour.

En dehors de cette évidence iconographique, il n'existe aucune littérature susceptible de nous informer sur l'histoire et les personnages de la tradition féminine à la cour. En fait, l'histoire de ces musiciennes fait l'objet de descriptions qui varient selon la période, le contexte culturel et les groupes ethniques auxquels appartiennent les interprètes. Les appellations les plus courantes sont: ganikā, naikin, kalavantin, lolonis, domines, kenchen, nautch girls, danseuses, ainsi que les termes modernes de tawā'if et bāijì. Bien que ces dénominations correspondent chacune à une catégorie spécifique d'artistes ${ }^{4}$, elles peuvent cependant être globalement regroupées en une seule typologie, en fonction de l'activité principale qui leur est commune, celle de divertir par leur musique et leur danse une audience masculine triée sur le volet.

7 En raison de cet aspect particulier de leur profession, ces artistes étaient souvent considérées comme de simples courtisanes, une catégorie sociale floue, qui inclut pêle- 
mêle concubines et musiciennes professionnelles et contribue ainsi à rendre ces dernières victimes de discrimination culturelle.

Ce mélange des rôles apparaît déjà dans le Kāma Sutra, traité sur l'art de l'amour sensuel écrit par Vatsyayana, au IV ${ }^{e}$ siècle de notre ère. Même si, dans la première partie de son ouvrage (Vatsyayana : 3-11), l'auteur insiste sur le fait que « les principes de l'art érotique doivent être transmis aux filles de rois et de ministres comme aux courtisanes ", c'est cette dernière catégorie qui l'intéresse particulièrement en tant qu'élément social et culturel important. En fait, les courtisanes appelées ganikā enseignaient l'art de la musique et du plaisir aux fils des princes et des nobles.

Les prostituées qui sont belles, intelligentes et instruites occupent une place d'honneur dans la société et sont connues comme courtisanes (ganikā). Elles sont respectées par les rois ; les gens honorables chantent leur louange ; respectées pour leur art, elles vivent exposées au regard de tous.

Selon la tradition transmise par Vatsyayana, les courtisanes doivent posséder, en plus de l'art érotique, soixante-quatre arts corrélatifs dont les plus importants sont dans l'ordre (Vatsyayana : 3-14) :

- 1. La musique vocale

- 2. Les instruments de musique (percussions, cordes, tambours et flûtes)

- 3. La danse (mouvements des mains et du corps, expression, émotion, sentiment)

- $[. .$.

- 27. L'art de jouer de la vina et du damaru

- 54. La science du dictionnaire

- 55. Les mètres poétiques

- 56. La versification et les formes littéraires.

Par ces brèves descriptions, Vatsyayana met en évidence les principaux traits qui caractérisent, à mon avis, la tradition féminine de la musique de cour indienne. En effet, en comparant ces passages du Kāma Sutra avec les documents écrits et les données que j'ai recueillies lors de mes recherches sur le terrain, j'ai pu identifier deux aspects importants, l'un social et l'autre artistique, qui m'ont permis de suivre la continuité historique de la tradition de cour depuis l'époque de Vatsyayana jusqu'à nos jours.

11 D'un point de vue social, selon l'auteur du Kāma Sutra, une courtisane qui a des talents artistiques (ganikā) est « une prostituée, belle, intelligente et instruite, qui occupe une place d'honneur dans la société».

12 La ganikā est donc une "femme publique " qui exerce son art en dehors de la sphère domestique tout en bénéficiant d'une position respectable au sein de la société. Cette situation n'est pourtant pas normale puisque, dans la société traditionnelle hindoue comme plus tard dans la société musulmane, les femmes sont reléguées à la vie domestique, et qu'elles ne sont pas autorisées à exercer une activité professionnelle en dehors du cercle familial (Altekar 1959, Gaur 1980). C'est pourquoi les musiciennes professionnelles étaient socialement assimilées à des concubines, alors même qu'elles suscitaient le respect et l'admiration par leur art (Moti 1973).

13 Leur lieu d'expression était effectivement les réunions d'hommes appelées goshti, puis, durant la période musulmane, mahfil, au cours desquelles les artistes dansaient en interprétant des chansons érotiques ou romantiques. Il est vrai que, dans un tel contexte, la grâce et la beauté jouaient un rôle très important ; cependant, on appréciait l'art et la compagnie de ces femmes surtout en raison de leurs manières raffinées, de leur instruction et de leur culture artistique, poétique et musicale (les soixante quatre-arts du 
Kāma Sutra). Ainsi, dès l'époque de Vatsyayana, un hiatus culturel séparait les professionnelles des femmes confinées à la sphère domestique.

D'après des sources plus récentes, la profession de musicienne et de danseuse de cour se transmettait traditionnellement par lignée matrilinéaire avec des rituels spécifiques ${ }^{5}$. Les filles apprenaient la musique et la danse de leur propre mère ou de musiciens de classe inférieure, et elles étaient engagées dans la vie professionnelle dès leur puberté, par une sorte de mariage symbolique qui les liait à la profession plutôt qu'à un seul homme. La cérémonie sanctionnait la défloration de la jeune fille qui accordait pour la première fois ses faveurs à son premier protecteur. Voici comment le savant indien Raghunathji (1884 : 171) décrit le rite nuptial en usage parmi les artistes de cour de la région de Maharashtra :

Le prix du dépucelage varie de cent à deux mille roupies, selon la beauté et les talents de la jeune fille. Le maître peut jouir de la compagnie de la fille pendant deux mois durant lesquels il doit lui offrir des vêtements, des bijoux et de l'argent. La célébration de l'événement commence huit jours avant que celui-ci ne devienne effectif. [...] Le maître se charge des frais de la fête. Cette nuit-là, la jeune fille, richement parée, se rend dans la maison de son maître en voiture, accompagnée d'un domestique ou d'une servante. Elle passe la nuit avec lui et revient le lendemain où l'attend un somptueux petit-déjeuner. A cette occasion, les amis proches sont invités. Ainsi se conclut la cérémonie.

Des rituels semblables étaient célébrés en grande pompe dans le nord du pays où le phénomène était également répandu. Un document du XVIII siècle mentionne une coutume particulière concernant la défloration de la jeune fille de la classe des tawā'if. La cérémonie du nath utāmā (ouverture de l'anneau de nez) sanctionnait le dépucelage de la fille tawä'if par son premier « client» (Maciszewsky 1998). L'anneau d'or ou d'argent qui ornait le nez était effectivement le symbole typique de la virginité de la jeune fille. Le fait de l'ôter ou de le remplacer par un bijou en perle ou en diamant représentait l'initiation de la fille aux activités musicales et sexuelles ${ }^{6}$.

Les tawā'if n'étaient pas autorisées à se marier officiellement; cependant, elles restaient souvent attachées à un seul homme qu'elles considéraient comme leur bienfaiteur. Ce rapport de fidélité durait parfois toute une vie et les artistes dépendaient de leur maître comme des courtisanes. Leur condition de concubine les distinguait des mères de famille ; mais en même temps, leur fidélité et leur loyauté à l'égard de leur protecteur les différenciaient des vraies prostituées qui, elles, étaient à la merci des clients de passage (Post 1988). Les enfants issus de ces couples non mariés étaient élevés par leurs mères ; celles-ci préparaient les garçons à suivre une carrière de musiciens accompagnateurs de courtisanes et les filles à devenir musiciennes et danseuses, formant ainsi une caste professionnelle autonome? ${ }^{7}$.

D'un point de vue artistique, les tawäif se distinguaient par leur maittrise de quelques-uns des soixante-quatre arts mentionnés par Vatsyayana et par leur aptitude à exprimer, grâce à un savant mariage du son et du mouvement, l'érotisme et le charme féminin.

Malgré le fait que ces artistes constituaient l'attraction principale des réunions d'hommes, la tradition classique de l'Inde s'est toujours désintéressée d'elles. En effet, celles-ci ne figurent dans aucune annale alors qu'elles sont mentionnées dans les récits des premiers voyageurs étrangers invités à ces manifestations musicales. Par exemple, F. Bernier, qui vivait à la cour de l'empereur Shah Jahan (1594-1666), décrit les réunions musicales animées par les artistes kenchen et porte un jugement favorable sur leurs remarquables talents de danseuses et de chanteuses. 
Celles que l'on appelle kenchen, les «dorées ", les «fleuries », celles-là, dis-je, étaient introduites dans le sérail du temps de Shah Jahan et passaient parfois toute la nuit à animer ce genre de fêtes en dansant et en chantant. Ces filles ne sont pas de cette sorte qui se prostitue à n'importe qui ; la plupart d'entre elles sont belles et bien mises et elles sont d'excellentes chanteuses et danseuses à la mode du pays, étonnantes par la souplesse de leur corps et l'agilité de leurs mouvements; et pourtant, elles se retrouvent au même rang que les filles publiques. (Bor 1987)

Les hôtes étrangers qui ne saisissaient pas toujours les subtilités musicales et linguistiques des chansons étaient pourtant frappés par le côté spectaculaire de ces divertissements exotiques. Durant la période coloniale britannique, les femmes artistes étaient désignées par les expressions génériques de « filles danseuses » ou "nautch girls » (de la racine natchna qui signifie "danser») par allusion à la partie dansée de leurs spectacles, termes qui ont longtemps occulté la contribution musicale de ces femmes ${ }^{8}$. En réalité les nautch girls n'étaient pas uniquement des danseuses, elles s'exprimaient à la fois par la danse et par le chant, témoignant ainsi de l'existence d'une forme esthétique ancienne et spécifiquement féminine. En outre, d'après la description de Vatsyayana, elles animaient les réunions masculines en récitant de la poésie et en offrant le raffinement et la grâce de leur compagnie. Selon des sources écrites et iconographiques ${ }^{9}$, certains des résidents coloniaux se mirent à imiter l'aristocratie indienne en appréciant les prestations musicales et dansées des nautch girls et en sollicitant leur présence et leurs faveurs dans leurs maisons. Dans son livre, Nevile écrit :

Les sahibs adoptaient avec bonheur les coutumes locales qui rendaient leur vie plus agréable, spécialement dans le domaine des loisirs et des plaisirs, où ils se délectaient en mâchant du bétel, fumant le hookah et appréciant la poésie persane en compagnie de la noblesse locale. Leur principale drogue était les nautch, sans lesquelles aucun divertissement pour Européens ne semblait complet. Tous les nouveaux arrivants étaient introduits à ces passe-temps pour hommes. Les memsahibs, qui jusqu'au début du dix-neuvième siècle ne partageaient pas les scrupules des générations postérieures et montraient du respect pour le style de vie indigène, assistaient librement aux fêtes nautch. Même après l'arrivée massive des dames anglaises et le changement de mode de vie, les sahibs préservèrent leur goût pour les nautch en tant que spectacle. C'était devenu une forme admise de divertissement et sa popularité restait intacte. (Neville 1996 : 49-52)

Alors que certains participaient avec enthousiasme aux soirées des mahfil, la majeure partie de la société coloniale (principalement anglaise) les condamnait avec sévérité. Pour l'éthique occidentale de l'époque, le spectacle des nautch girls posait un problème délicat. Ceux qui ne comprenaient pas les nuances linguistiques des chants ou les figures expressives et rythmiques raffinées des danses, et se bornaient aux aspects extérieurs des interprétations et au charme physique des artistes, considéraient ces représentations comme lascives et trop sensuelles pour la morale anglaise. Dans des articles parus à la fin du XIX ${ }^{\mathrm{e}}$ siècle puis rassemblés et publiés par la Christian Literature Society en 1893, nous pouvons lire à ce sujet :

Chaque fois qu'un vice-roi ou un gouverneur est en tournée officielle, il est diverti à maintes reprises par des femmes qui mènent la plus honteuse des vies. Les épouses de ces hauts fonctionnaires sont également présentes pendant que leurs sœurs dépravées dansent et chantent pour leur amusement. (Tiré de The Harvest Field) Que les épouses anglaises se mettent à la place de leur sœurs mariées hindoues. Que ressentiraient-elles si, nuit après nuit, leurs maris les abandonnaient pour rejoindre des prostituées? (Extrait de Partial Social Reform in India Through the Influence of European Ladies) 
21 En Inde du Sud, les colons lancèrent, dès 1892, un mouvement pour abolir les spectacles des nautch girls, prétendument responsables de crime et de prostitution, condamnant indifféremment les devadāsī du Sud et les traditions courtisanes du Nord. Alors que l'origine et l'évolution de la tradition féminine de cour restent encore obscures, il est évident que sa fin a été précipitée par cette formidable pression qui visait à son abolition.

Peu après, le mouvement se transforma en une campagne qui couvrit tout le souscontinent, du Sud au Nord, et fut soutenue à la fois par les derniers souverains indiens, maintenant affaiblis sous le joug britannique, et par l'intelligentsia bourgeoise, désireuse de voir l'Inde débarrassée de ses structures sociales traditionnelles. Des journaux indiens de l'époque rapportent :

Il est temps que les classes officielles anglo-indiennes comprennent que les nautch ne sont pas des divertissements aussi innocents qu'on le leur a fait croire jusqu'ici. Je souhaite que le vice-roi et les gouverneurs de province soient les premiers à servir d'exemples dans ce domaine, en refusant d'assister à des fêtes dans lesquelles les nautch constituent une des principales attractions. Pour l'amour de tout ce qui est pur, les nautch girls devraient être bannies de la société respectable. (Indian Spectator, Bombay, 1893)

Dans un fameux rapport intitulé "Opinions sur la question des nautch », établi par la Punjab Purity Association (1894), des membres de l'aristocratie indienne émettent des jugements très fermes sur l'abolition de l'institution des nautch girls :

Cette hideuse femme danse; et elle sourit en dansant; et elle coulisse des regards furtifs! En apparence une douce demoiselle, une personne charmante. Mais, qu'estce qui se tapit sous cette belle enveloppe ? Une férocité infernale. L'enfer brille dans ses yeux. Dans sa poitrine bouillonne un vaste océan de venin. Autour de sa charmante taille vivent les furies de l'enfer. Ses mains brandissent d'invisibles poignards toujours prêts à frapper les victimes ignorantes ou volontaires qui tombent sur son chemin. Ses flatteries sont la ruine de l'Inde. Hélas! Son sourire est la mort de l'Inde.

Presque toutes les nautch girls sont des prostituées en Inde. Quelle autre jeune femme respectable se mettrait à danser et à chanter devant un public pour de l'argent? C'est parce qu'elles sont les dépositaires de notre musique qu'elles réussissent à faire autant de mal. L'union de la séduction féminine et du charme de l'art musical les rendent attrayantes aux yeux d'une jeunesse inconsciente. Pendant les fêtes nautch, les jeunes gens sont pratiquement introduits dans la société de ces femmes de mauvaise réputation. Cela les entraîne à leur rendre secrètement visite dans les bordels. Ainsi, les jeunes gens sombrent peu à peu dans le vortex de la fornication.

24 Cette attitude sévère que les Indiens adoptèrent à l'égard d'une tradition autochtone découlait d'une série de causes occasionnées par les profonds changements sociaux qui marquèrent la fin du XIX siècle. L'influence politique et culturelle grandissante des colonisateurs britanniques allait de pair avec la montée des classes bourgeoises et le déclin du monde des cours et de leurs traditions artistiques particulières, soumises à un inévitable processus de changement et, souvent, de déclin. Quant aux chanteuses et aux danseuses de cour, faute de fêtes somptueuses organisées par l'aristocratie, elles manquèrent d'espace dès la fin du XIX ${ }^{e}$ siècle et leur tradition, en se corrompant, se rapprocha de plus en plus de la prostitution.

Cependant, comme nous venons de le voir, il existait une certaine conscience du fait que les nautch girls étaient les dépositaires d'un répertoire musical et que leur héritage artistique méritait d'être protégé. Dans un article de la Christian Literature Society (1893) de Madras, on trouve la citation suivante : 
Aux femmes nautch elles-mêmes: nous sommes tenus d'œuvrer pour leur régénération. En les protégeant, peut-être même pour leur musique, nous leur accordons position et prééminence dans la société... survenus dans l'ensemble du marché de production et de consommation de la musique qui entraînèrent la chute de la classe des musiciennes et des danseuses professionnelles (Cassio 2001). La tradition se tourna en partie vers les industries en pleine expansion du cinéma et de la musique commerciale et s'adapta aux nouvelles exigences du marché, qui était avant tout à la recherche de chanteuses et de danseuses capables de jouer à l'écran ou d'enseigner de façon professionnelle (Lakshmi 2000). Mais, sous le choc de l'humiliation, certaines de ces femmes interrompirent leurs activités artistiques et rompirent définitivement les liens qui les rattachaient à la tradition.

Plusieurs intellectuels de l'époque s'efforcèrent de préserver les répertoires musicaux en les isolant de leur contexte, en les séparant de leur fonction de divertissement pour hommes et de prestations d'artistes de cour ; ils décrétèrent ainsi la création de nouvelles formes artistiques et de nouveaux modes d'expression. Cette démarche donna naissance à la danse du Bharatantyam, qui quitta l'espace sacré des temples pour rejoindre les scènes publiques; elle fut également à l'origine des concerts modernes de musique classique dans lesquels il était interdit d'accompagner le chant par la gestuelle (Kersenboom 1987, Gaston 1996). courtisanes dansaient et chantaient furent formellement interdites. La tradition survécut de façon clandestine pendant une longue période, grâce au soutien de la classe prospère des hommes d'affaires indiens, plus intéressés par l'aspect physique que par le plaisir intellectuel des représentations.

le désir de " purifier » les anciens systèmes sociaux et de faire pièce à la corruption de la société indienne, combattit encore plus vigoureusement le phénomène des nautch girls qui continuait à sévir dans les grandes villes, notamment à Delhi, Bombay, Calcutta, Lucknow et Bénarès. Elle tenta d'éradiquer cette ancienne coutume par une brutale répression policière. A titre d'exemple, rappelons cette nuit de décembre 1958 à Lucknow où la police ferma de force tous les locaux des courtisanes et arrêta un grand nombre d'entre elles (Kippen 1988). Cela se produisit également dans d'autres villes où les quartiers réservés traditionnellement aux maisons des nautch girls furent graduellement dépeuplés ${ }^{10}$.

Il en résulta que très peu de femmes artistes survécurent à la révolution culturelle du $\mathrm{XX}^{\mathrm{e}}$ siècle. Dissimulées dans les ruelles de la cité, certaines continuent cependant à chanter et à danser pour quelques hommes d'affaire fortunés ou pour animer des fêtes privées, des mariages, des naissances ou d'autres célébrations. A l'époque moderne, on appelle ces femmes tawä'if et bāijīi, termes péjoratifs dans leur acception courante, autrefois simples euphémismes pour désigner les «concubines» ou les "prostituées». Dans les paragraphes qui suivent, je tenterai de souligner les caractéristiques de leur tradition artistique en m'appuyant sur les sources historiques modernes ainsi que sur mes propres observations de terrain. 


\section{Tawā'if et bāìīi}

31 Tawā'if et bāijī sont des appellations courantes pour désigner les artistes de cour aux XIX et $\mathrm{XX}^{\mathrm{e}}$ siècles. Ces termes comportent d'importantes nuances dans leur sens et leur pertinence culturelle. Ils méritent d'être commentés ici. En nous appuyant sur les témoignages d'un passé récent et sur la contribution d'artistes vivants, nous pouvons analyser certains aspects de l'esthétique et de l'art féminins de la musique restés dans l'ombre jusqu'à présent.

32 La catégorie des tawā'if recouvrait une grande palette d'artistes et de courtisanes qui peuplèrent les cours princières dès le XVIII ${ }^{\mathrm{e}}$ siècle. Le phénomène était spécialement lié au développement des cours musulmanes de l'Inde du Nord, parmi lesquelles les centres de pouvoir de Delhi et de Lucknow revêtaient une importance particulière. Avec le déclin de l'Empire Moghol, la ville de Delhi perdit progressivement de son importance et une partie de la scène politique du XVIII ${ }^{e}$ siècle se transféra à Lucknow, siège des nawabs d'Oudh, où se rendirent de nombreux artistes de la cour de Delhi. C'est là que la culture des réunions musicales animées par les tawā’if atteignit l'apogée de sa splendeur, sous le patronage de souverains et de gentilshommes amoureux de l'art (Sharar 2001).

Les nobles et les riches confiaient leurs fils à ces dames accomplies pour apprendre l'étiquette et l'art de la conversation. Wajid Ali Shah, le dernier nawab d'Oudh dont le règne est évoqué comme l'âge d'or de la musique et de la danse, alla même jusqu'à fonder une institution appelée "Parikhana » (maison des fées), où plus de cent filles apprenaient la musique et la danse. Certaines d'entre elles furent introduites au palais comme begums. (Nevile 1996 : 39)

Selon une des dernières protagonistes de cette tradition ${ }^{11}$, le terme tawā’if, dérivé du persan, littéralement "celle qui se déplace ", suggère le mouvement de la danse dans l'espace (Nevile 1996 : 98) aussi bien que le déplacement à l'intérieur de la ville ou dans le pays, pour donner des représentations. Il existait même une catégorie de tawā’if appelée deredar (dera signifiant «tente »), des artistes itinérantes qui suivaient fidèlement leurs souverains et campaient avec eux pendant leurs longues expéditions vers de lointains champs de bataille. Selon Sharar, cette pratique fut introduite par le nawab shuja ud Daula qui avait l'habitude d'emmener sa musicienne favorite dans sa suite, pendant ses longs voyages.

Lorsque le nawab faisait le tour de ses provinces ou voyageait, leurs tentes étaient chargées avec pompe sur des chars à bœufs, en même temps que celles du souverain, et elles étaient gardées par des compagnies de dix à douze soldats. Les riches et les chefs se mirent eux aussi à adopter ouvertement cette coutume royale, en se faisant accompagner par les courtisanes pendant leurs voyages. (Sharar 2001 : 35)

En tant que classe, les tawā'if étaient bien éduquées, raffinées, et elles faisaient partie des rares membres de la société indienne qui savaient lire, écrire, apprécier la poésie et composer des vers.

Les musulmans plaisantent en disant qu'en matière de fidélité, la danseuse dépasse l'épouse. [...] Pleine d'esprit, elle est généralement plus cultivée que la femme mariée. La fascination qu'elle exerce est due principalement au fait que, dans un pays où, pour des raisons de convenance, l'épouse n'est pas admise en société, elle constitue la plus charmante et la plus agréable des compagnies. (Raghunathji 1884 : 174) 
C'est pour toutes ces raisons que les tawāif étaient non seulement respectées et aimées par leurs protecteurs, mais également employées dans les familles royales et chez les nobles pour enseigner les bonnes manières. Saira Begum, une artiste contemporaine descendant de la tradition de cour, dit à ce sujet :

Le terme de tawāif désigne aussi les personnes qui enseignaient comment il fallait s'asseoir, saluer les gens, écouter pendant les mujras ${ }^{12}$, parler et chanter. Avant, quand nous avions encore des rois, les tawä'if leur apprenaient comment ils devaient se comporter en différentes situations ${ }^{13}$.

Les tawäif pouvaient se mettre à leur compte, mais elles organisaient souvent leurs

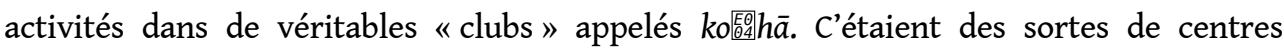
culturels animés par des soirées de musique et de danse (mahfil), auxquelles participaient parfois des poètes éminents, des artistes, des hommes de lettres et des politiciens ${ }^{14}$.

Les maisons de Chaudrayan Haidar Jan et de quelques autres courtisanes de haute volée étaient des «clubs» pour personnes distinguées. L'influence britannique changea la situation de sorte que, malgré l'apparition de nouvelles formes d'inconduites sociales, les gens ne voulurent plus fréquenter ouvertement les maisons des courtisanes et profiter du plaisir de leur compagnie. (Sharar 2001 : 196)

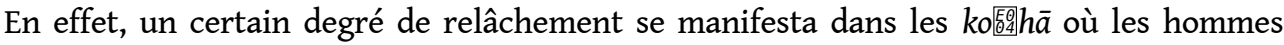
purent s'adonner à des plaisirs demeurés tabous aux yeux de la société indienne, en

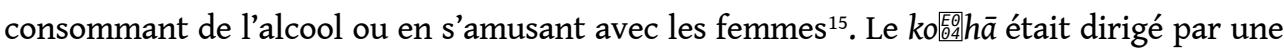
vieille et célèbre tawā'if qui recrutait de jolies jeunes filles et leur enseignait la danse, la musique et les bonnes manières. C'était un honneur pour la tawāif d'appartenir à une famille (khandan) d'artistes renommés.

De nombreuses tawā'if étaient d'extraordinaires artistes dont le talent était hautement récompensé par leur protecteur, ce qui les rendait célèbres et très riches. Pour un homme, le fait d'avoir une relation avec une tawā'if n'était pas signe de vice et de honte, mais symbole d'une position économique et sociale élevée.

A Lucknow, la fréquentation des courtisanes commença sous le règne de Shuja ud Daula. Il était de bon ton pour un noble de sortir avec quelque beauté de bazar, pour le plaisir ou pour la distinction sociale. Un homme cultivé tel que Hakim Mandi, devenu plus tard vazir, dut ses premiers succès à une courtisane nommé Piyaro qui lui avait avancé de l'argent pour lui permettre d'offrir un présent au souverain lors de sa première nomination au poste de gouverneur de la province d'Avadh. [...] Même si ces pratiques eurent un effet dégradant sur la moralité, elles améliorèrent en même temps les manières et le raffinement. (Sharar 2001 : 192)

Bien qu'elles aient été d'agréables animatrices, les tawāif était surtout spécialisées dans l'art d'interpréter des pièces de musique en dansant et chantant simultanément avec une sensualité particulière (bhāv), ce qui les amenait à utiliser tout leur corps comme un instrument. Maciszewsky (1998 : 118) écrit à ce propos :

Avec son corps, elle illustrait la poésie d'un bandish (bhāv batānāa, littéralement « dire la sensation »), spécialement par l'adā (mouvements des mains et du torse) et le $h \bar{a} v$ bhāv (expression du visage). Parfois elle dansait le tatkār (travail du pied) pendant que ses accompagnateurs jouaient des interludes instrumentaux.

Les musiciens qui accompagnaient le spectacle de la tawä'if jouaient du $\operatorname{tablä}^{16}$ et du sārangi ${ }^{17}$ et recevaient de ses mains leur seule source de revenus. A certaines occasions, la tawāif présentait elle-même les musiciens à la cour où ils se faisaient remarquer et apprécier :

De la période nawabi jusqu'au début $\mathrm{du} \mathrm{XX}^{\mathrm{e}}$ siècle, les musiciens dépendaient fortement des tawā'if, non seulement pour leur patronage direct, mais aussi pour 
leur introduction à la cour ou dans les maisons des riches aristocrates. (Kippen

$1988: 23)$

41 d'attraction des réunions pour hommes, appelées soit mahfil (ou ba臤a mahfil) lorsqu'il y avait beaucoup d'hôtes, soit mujra lorsqu'un nombre restreint de spectateurs assistaient à une représentation privée. En plus de son cachet pour la soirée, l'artiste recevait parfois des présents et de l'argent que les spectateurs lui donnaient en guise d'appréciation. Elle payait alors les musiciens, en gardant la plus grande partie de l'argent pour elle-même. Les relations entre les tawāi if et leurs accompagnateurs étaient cependant compliquées du fait que les musiciens étaient souvent leurs maîtres. En effet, tandis que l'artiste apprenait de sa mère l'art de chanter et de danser avec grâce, c'est auprès des musiciens plus âgés qu'elle s'initiait aux répertoires de la musique et de la danse. Les mentors et maîtres de musique des tawāì étaient le plus souvent des joueurs de sārangī, instrument dont le timbre rappelle la voix humaine et qui était utilisé pour enseigner les airs et les ornementations vocales ainsi que pour servir d'accompagnement pendant les concerts, comme voix de soutien.

Lorsqu'une tawā'if atteignait une position honorifique dans le domaine artistique et social, ou qu'elle prenait de l'âge, elle était appelée begum, si elle était musulmane, ou baìiji, si elle était hindoue, les deux appellations respectueuses signifiant "madame ", sans spécification professionnelle ou artistique particulière. Lorsque la bataille contre les nautch girls fit rage dans toute l'Inde, les tawā'if reçurent un coup terrible en tant qu'institution, et plusieurs artistes se firent promouvoir au rang de begum pour échapper aux accusations de prostitution ou encore se firent appeler par le terme générique de bāiji (sans tenir compte des facteurs ethniques ou religieux) pour établir une distinction entre les artistes et les prostituées.

Même de nos jours, le terme de tawā'if est généralement utilisé comme synonyme de prostituée alors que l'expression plus neutre de bāijì rappelle le monde des artistes courtisanes. C'est pour cette raison que je préfère utiliser ce dernier terme pour parler de la tradition des dernières chanteuses de cour.

\section{L'esthétique du chant bāîji à travers une analyse de la gestuelle}

Les bāijī représentent le chapitre final de l'histoire des artistes de cour et fournissent le dernier exemple d'une esthétique musicale féminine consciente.

Grâce à mes recherches dans la région de Bénarès ${ }^{18}$, j'ai pu identifier et documenter ce phénomène à travers le témoignage de plusieurs interprètes contemporaines. Je me suis particulièrement intéressée à l'artiste Saira Begum, choisie pour ses talents vocaux exceptionnels et son répertoire musical riche en compositions très anciennes, restées pratiquement inconnues jusqu'à nos jours. J'ai travaillé avec elle pendant plus de trois ans, conservant constamment mes leçons et mes discussions quotidiennes avec elle. Par la suite, j'ai comparé cette documentation avec des sources écrites et iconographiques ainsi que les matériaux audio et vidéo disponibles.

L'observation et la participation m'ont permis de mettre en évidence certains faits encore peu connus sur cette tradition. C'est eux que j'aimerais exposer dans les pages qui 
suivent. La première observation importante concerne la nature spécifique du répertoire bāijī qui comporte deux éléments principaux :

- des compositions mélodiques et poétiques avec un contexte érotique (chants d'amour);

- l'expression des sentiments féminins à travers les textes musicaux, par le biais de la danse et du chant.

Un autre trait marquant de ma recherche sur le terrain fut la découverte de la large palette des formes musicales interprétées par les bāijīi, du rāga ${ }^{19}$ classique à la musique dite semi-classique et à la musique populaire. Le talent de l'artiste est donc évalué en fonction, non seulement de ses aptitudes vocales, mais également de sa versatilité et de sa capacité à offrir des programmes hétérogènes comprenant différentes styles de musique et de danse.

En fait, comme me l'expliqua Saira Begum, l'aspect riche et multiforme du répertoire découle de l'apprentissage musical et culturel composite des bāijī. En règle générale, ces artistes sont issues des régions rurales et apprennent leur répertoire populaire dans leur environnement familial. C'est seulement après leur établissement en ville qu'elles acquièrent une sorte de savoir musical et d'entraînement vocal auprès des maîtres de la musique classique, en étudiant les formes complexes de l'improvisation virtuose sur les principales mélodies des rāga ainsi que les formes de la musique semi-classique. Malgré leur formation musicale hétérogène, leur répertoire principal consiste en différentes

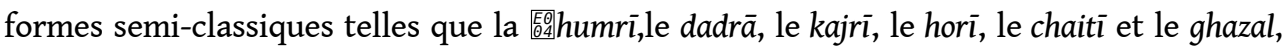
dans lesquelles des éléments de musique classique se mêlent à ceux de la tradition populaire.

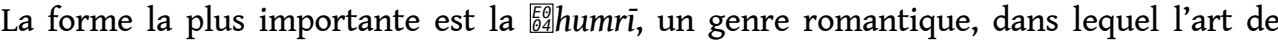
l'interprète réside dans la répétition du même fragment d'un petit texte érotique avec de

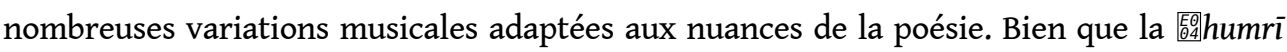
ait fait l'objet d'études récentes (Ganguly 1987, Manuel 1989) ${ }^{20}$, la manière dont les bāiji donnent à cette musique une caractéristique spécifique, allant jusqu'à créer une forme séparée, est encore peu connue. Dans l'interprétation des bāijīi, en effet, la musique est accompagnée de gestes des bras et d'expressions faciales ou d'une danse dans laquelle les expressions aussi bien que la chorégraphie s'efforcent de rendre le sens des paroles.

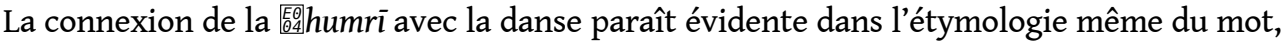
qui est dérivé de deux racines verbales 㢣humak (danser) et rizhana (divertir) (Banerjee 1986). Bien que les érudits restent partagés sur les origines de la 鄫humrī, il est certain que ce genre fleurit au XVIII siècle dans les somptueuses cours de la région de Lucknow et de

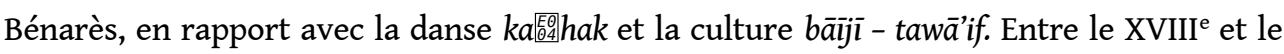
$\mathrm{XX}^{\mathrm{e}}$ siècles, la 粼humri fut donc la principale forme d'expression et le véhicule des divertissements dansés et chantés des artistes de cour, héritiers d'une tradition qui s'est perpétuée, au moins théoriquement, de Vatsyayana jusqu'à nos jours. 
Fig. 2 : Saira Begum en train de chanter et de danser.

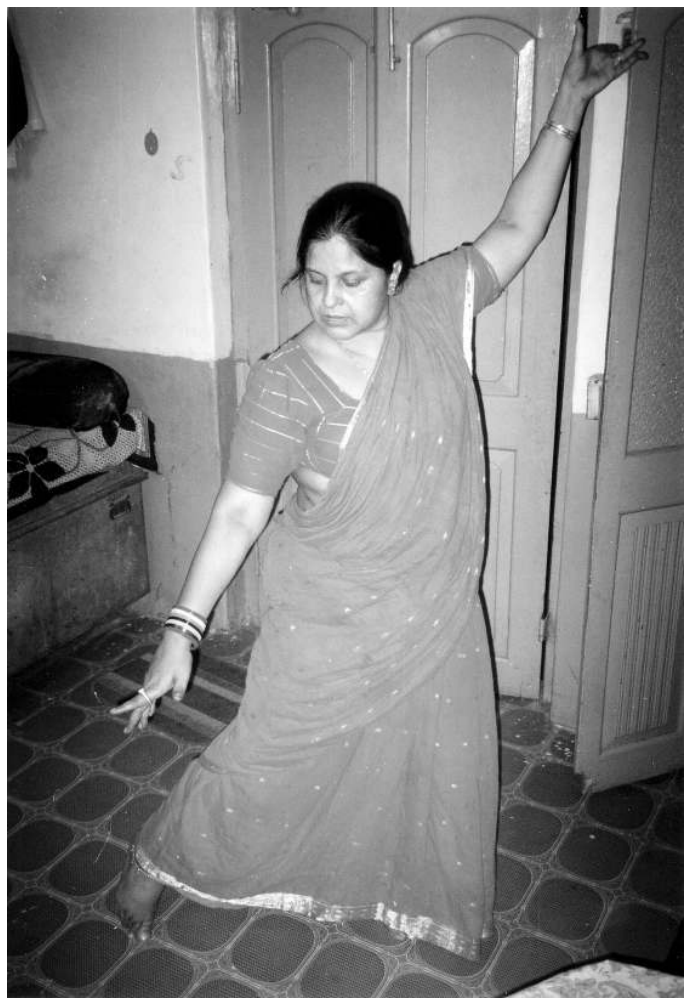

Photo F. Cassio

Fig. 3 : Saira Begum interprète le même chant debout et assise.

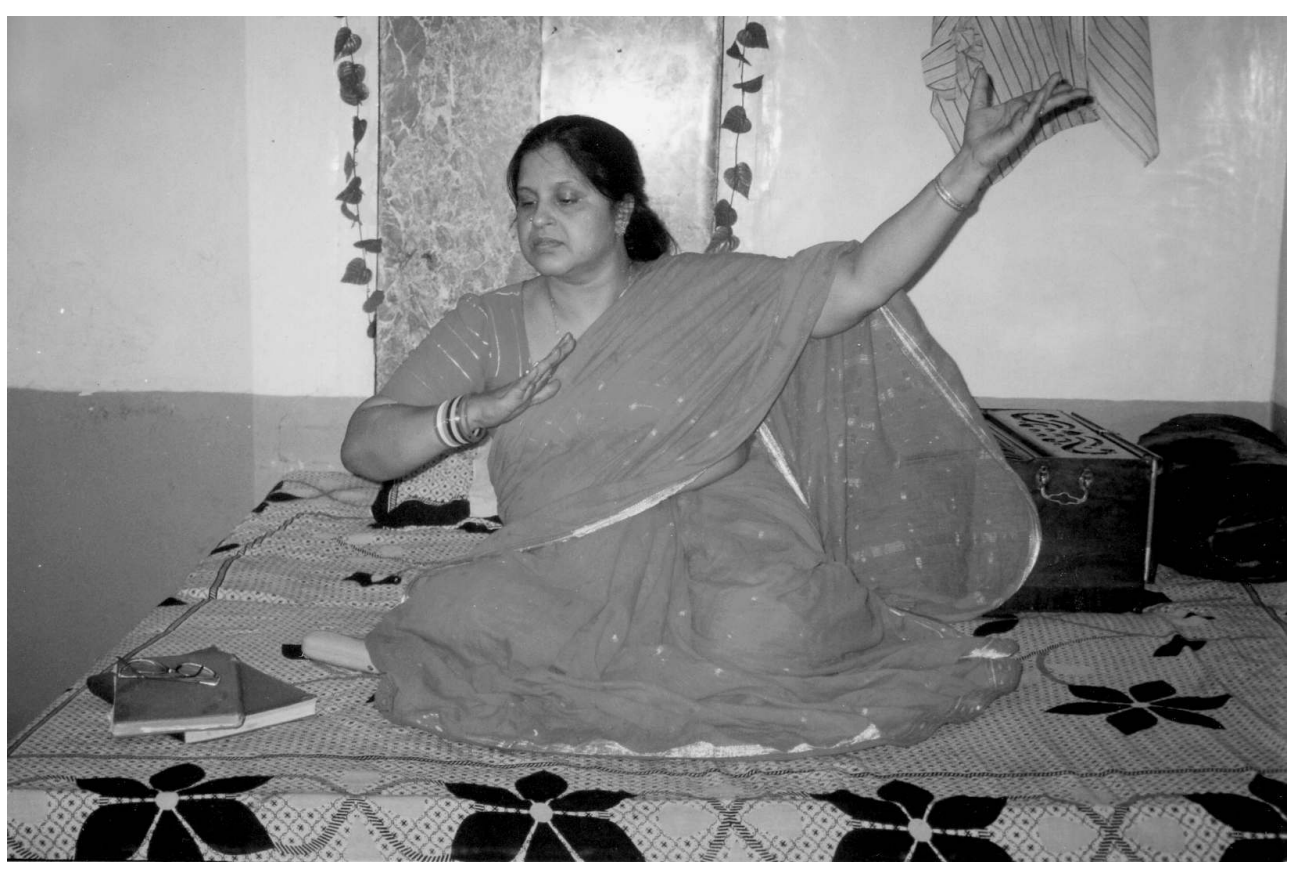

Les photos 2 et 3 illustrent toutes deux le mot bijeli qui signifie « tonnerre » et que Saira évoque par un large mouvement de bras. Photo F. Cassio 
Grâce au témoignage de Saira Begum et d'autres bāijīi que j'ai fréquentées pendant des années, j'ai constaté que l'interprétation du chant semi-classique (notamment la 熙humrī et le $\mathrm{dadra \overline {a }}$ obéissait à des règles précises, dans lesquelles la composante de la danse apparaissait sous deux forme :

- debout, selon une chorégraphie authentique qui exalte autant la narration du texte que la virtuosité de la danse elle-même (tatkar);

- assis, (betke), dans laquelle les variations musicales (appelées bolbanao) prédominent, utilisant seulement les gestes du bras (abhinaya) et les expressions du visage (hāv bhāv) :

De même, en se basant sur l'analyse textuelle de la 㯺humrī, Du Perron (ITC 2003 : 14) a

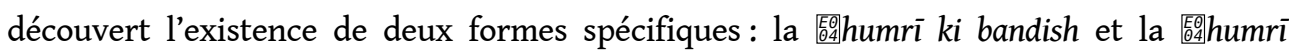
bolbanao, selon la prédominance de la danse ou de l'élément vocal.

Au niveau de la danse et de la musique, en règle générale, la forme dansée favorise le côté spectaculaire de l'événement en jouant sur les variations chorégraphiques, alors que la performance betke, plus raffinée musicalement et plus méditative, exalte les talents vocaux et le sens de l'improvisation de l'artiste en interprétant des variations musicales impromptues sur la mélodie de la composition de base. Ainsi, les variations de la danse l'emportent dans le premier cas, alors que celles de la musique prédominent dans le second. transmet un message érotique à l'auditeur. En réalité, ce n'est ni le texte poétique en luimême, ni la musique, ni la gestuelle empruntée à la danse classique kathak qui crée cette atmosphère troublante, mais plutôt la combinaison raffinée de ces trois langages.

Il est intéressant de noter à ce propos que les bāijì apprennent le chant classique (dhrupad ou khyāl $)^{21}$ avec les maîtres de musique et, séparément, la danse kathak avec les maîtres de la tradition de la danse. Par conséquent, il incombe aux bāīji elles-mêmes de combiner les expressions de la voix et de la danse, dans une démarche de créativité personnelle. Pour ce faire, la bāijì utilise un miroir pour étudier la gestuelle (abhinaya) empruntée au répertoire du kathak, qui convient le mieux à l'expression d'un chant particulier. Voici ce que Saira Begum dit à ce sujet ${ }^{22}$ :

Les yeux, la bouche... la façon de regarder, de sourire, de paraître triste, lentement, lentement on essaie et on crée son style. Le miroir est très important: lorsque je danse debout, je surveille ma posture: de cette façon? Non, ah! Cela va mieux. Puis, lorsque je fais le riyaz ${ }^{23}$ pour le chant, je m'assieds devant le miroir et j'observe mon visage : comment est-il ? Comment le rendre plus agréable, le nez comme cela, les yeux, les joues, la bouche : tout doit être « accha »... juste.

Saraswati Devi, une bāijī de Bénarès dit de même ${ }^{24}$ :

Conformément à l'enseignement de mes maîtres de chant et de danse, je construis mon répertoire et, avant de me présenter en public, je redis plusieurs fois, seule devant le miroir, la même phrase d'une 原humrī; je répète des dizaines de fois les mêmes mots avec des expressions différentes... 
59 que c'est ce phénomène de fusion entre la musique et le geste qui constitue la caractéristique majeure de la tradition bāijì et distingue celle-ci des autres formes contemporaines de la musique et de la danse en Inde.

Un examen approfondi de la gestuelle des bāiji révèle non seulement le caractère unique d'un art en voie d'extinction, mais aussi la marque profonde d'une esthétique consciemment féminine que plus personne ne connaît. La comparaison entre les gestes des artistes courtisanes et ceux des chanteurs de dhrupad et de khyāl montre en effet deux perceptions totalement différentes de l'interprétation musicale.

61 Comme on le sait, les chanteurs du répertoire classique accompagnent aussi leur chant de larges mouvements des bras, mais ce genre de gestuelle a une connotation profondément différente de l'abhinaya des bāìjī. En réalité, de tels gestes appartiennent à un système d'expression qui diffère en intention et en technique du langage gestuel du kathak auquel appartiennent les bāijìi. Les gestes des musiciens classiques sont enracinés dans l'instinct et la nécessité d'utiliser le mouvement pour donner de l'énergie à l'expression vocale. Voici ce que m'expliqua le maître du dhrupad, Ustad Rahim Fahimuddin Dagar, lors d'un entretien que j'ai eu avec lui à ce sujet ${ }^{25}$ :

En règle générale, les hommes font des mouvements lorsqu'ils chantent, chacun à sa manière. Aujourd'hui, la plupart des chanteurs le font fortuitement, suivant leur instinct. Celui qui bouge instinctivement essaie de suivre le son, mais il le fait par hasard parce qu'il ne dispose d'aucun système de référence. Dans le dhrupad, tel qu'il a été transmis par le gharānā des Dagar, nous avons le nāda yoga, un système réellement scientifique qui relie notre corps, nos actions et la production du son.

Dans le chant de Ustad Rahim Fahimuddin Dagar, chaque geste a sa raison d'être ; il vient de la tension interne du corps pour produire le son vocal dans ses nuances les plus complexes, dans le vrai style Dagar (Cassio 2000). Spécialement pendant le long ālāp (introduction du rāga), dans lequel le chanteur de dhrupad improvise en vocalisant une mélodie sans mots, la qualité du son et la manière de le produire doivent avoir un impact tel qu'il déclenche un effet émotionnel chez l'auditeur. La qualité du son et la puissance de son impact résultent d'une étude spécifique de la production de la voix et de la relation énergétique entre la voix et le corps, dans laquelle le geste n'est qu'une expression externe et, parfois, un moyen. 
Fig. 4 : Ustad Rahim Fahimuddin Dagar en train de chanter.

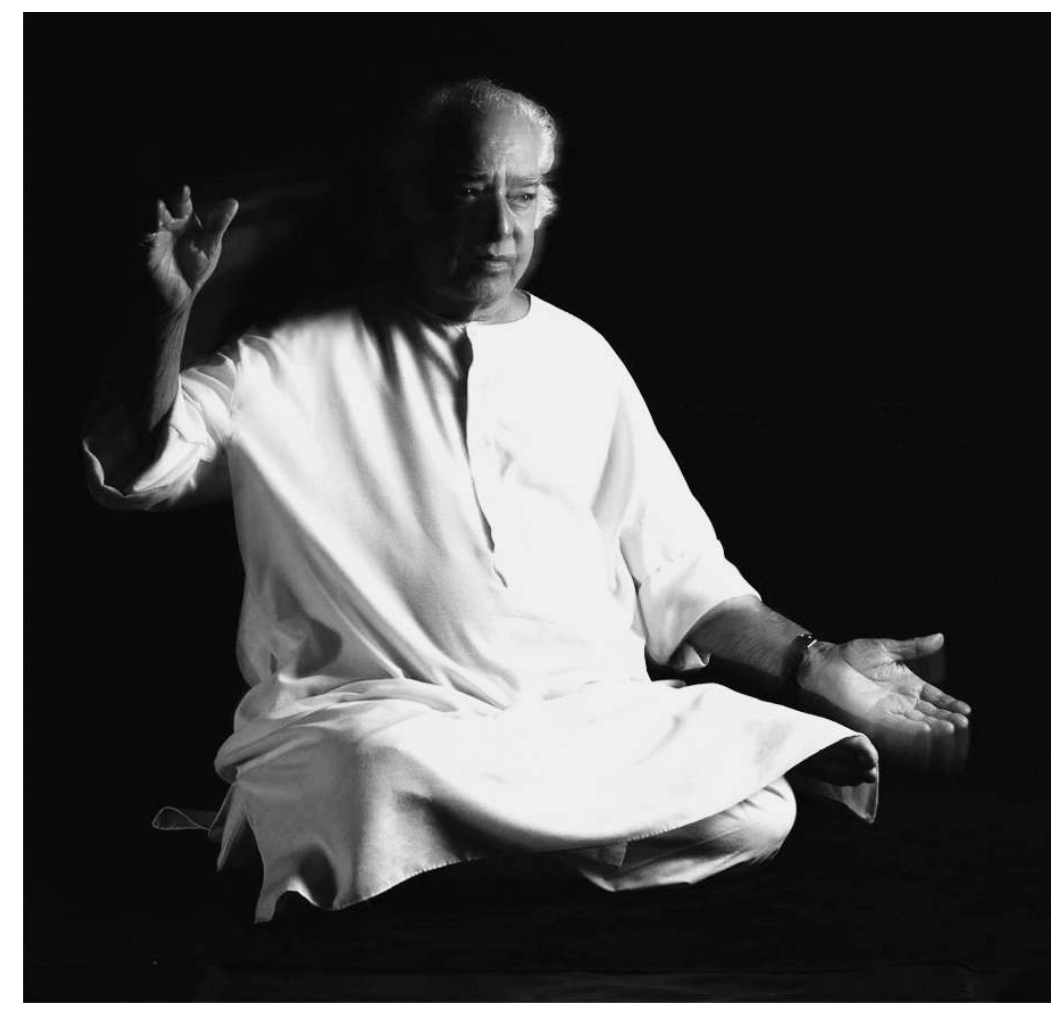

Photo Sarabjit Babra avec l'aimable autorisation de Bhai Baldeep Sing

Dans ce contexte, la gestuelle n'est pas une danse codifiée mais une adaptation instinctive $\mathrm{du}$ corps à la respiration du chanteur et aux poussées énergétiques de la tension expressive. En particulier, comme le pense le maître, les actions doivent s'accomplir conformément au nadā yoga, un système qui exploite effectivement les poussées énergétiques à l'intérieur du corps, la respiration et la production de son vocal, en accord avec la théorie du yoga. fondamentale entre la tradition bāijì et la musique classique. Si la gestuelle des bāīì a pour but la communication érotique subconsciente, les gestes des musiciens classiques sont, quant à eux, une représentation instinctive des mouvements du son à l'intérieur du corps. L'abhinaya révèle une réelle esthétique de la sensualité féminine alors que les gestes des musiciens expriment une sorte de prière intérieure, dont le sens est non seulement spécifiquement masculin (interprété par des hommes), mais également profondément mystique.

On pourrait se demander pourquoi les femmes n'ont jamais eu le droit de participer à cette forme de mystique musicale pour être simplement reléguées au rôle de séductrices. J'ai interrogé Ustad Dagar et d'autres musiciens de la tradition classique sur les raisons de cette interdiction. Au moins en ce qui concerne le dhrupad, ils m'ont répondu unanimement que le problème n'était pas d'ordre social mais "physique " : c'était une question d'énergie, puisque dans le chant dhrupad, l'implication du corps avec ces poussées d'énergie est d'une importance fondamentale; or l'énergie (kundalini) circule différemment dans le corps de la femme que dans celui de l'homme ${ }^{26}$ : 
Les vigoureux mouvements provoqués par la pratique du nāda yoga pourraient endommager la matrice de la femme, qui est destinée à donner la vie et doit, de ce fait, être protégée.

femme liée à ses devoirs domestiques et s'efforcèrent de participer à la culture et à la société de leur temps. Cependant, les premières grandes chanteuses de musique classique venaient de la tradition bāijī elle-même, comme l'indiquent leurs noms : Begum Akhtar, Kesarbai, Rasoolangai, Hirabai, Menakabai, etc. "modernes» de musique classique ont dû non seulement imiter servilement les interprètes masculins en ne laissant aucune place à leur propre créativité, mais elles ont également rejeté entièrement la tradition féminine passée, en évitant toute gestuelle qui rappelle le souvenir des bāiji. Ainsi, encore aujourd'hui, beaucoup d'entre elles évitent toute gesticulation pendant leurs interprétations, de peur que leur chant ne comporte la moindre allusion, même voilée, à la sensualité.

Bien que la tradition des bāijī soit vouée à la disparition avec cette dernière génération, un sentiment généralisé de honte face à ce phénomène est maintenant fortement enraciné en Inde, empêchant la récupération d'une part importante de l'histoire sociale et musicale du pays. Ce sentiment empêche également la reconnaissance d'une esthétique de chant spécifiquement féminine, transmise depuis l'époque du Kāma Sutra jusqu'aux bāijì modernes, comparable en quelque sorte à la logique, à la fonction et à l'esthétique de la musique classique.

72 La mythologie indienne raconte que la divinité de Shiva est incomplète sans son double féminin, sa shakti. Paraphrasant cette légende, nous pourrions dire qu'une histoire de la musique indienne dans laquelle seuls les musiciens mâles sont mentionnés est partiale et lacunaire : il lui manque la contribution des femmes à la culture musicale, laissée pendant des millénaires dans l'obscurité et dans l'oubli. 


\section{BIBLIOGRAPHIE}

ALTEKAR A. S. 1959 The Position of Women in Hindu Civilization. Delhi:Motilal Banarsidas Publishers.

BAHADUR Krishna 1998 Mira Bai and her Padas. New Delhi:Munishiram Manoharlal Publishers.

BANERJEE Projesh 1986 Dance in Thumrī. New Delhi:Abhinav Publications.

BOR Joep 1987 The Voice of the Sarangi. Bombay: National Centre for the Performing Arts.

BURCKHARDT QURESHI Regula 2001 « In search of Begum Akhtar: Patriarchy, Poetry, and

Twentieth-Century Indian Music », The World of Music 43/1: 97-137.

CASSIO Francesca 2000 Percorsi della voce. Storia e tecniche esecutive del canto dhrupad nell'India del nord. Bologna : Ut Orpheus. 2001« La musica classica indiana : mutamenti nella produzione e nella fruizione dopo il 1947 », in Atti del Congresso Italindia. Milano: Franco Angeli.

ERDMAN Joan 1992 Arts Patronage in India. New Delhi:Ajay Kumar Jain Manohar Pub.

GANGULY Rita 1987 « The Tradition of Thumri »,in Aspects of Indian Music - a Collection of Essays, edited by Sumati Mutatkar. New Delhi: Sangeet Natak Akademy.

GASTON Anne-Marie 1996 Bharata Natyam. From Temple to Theatre. New Delhi: Manohar Publishers. GAUR Albertine 1980 Women in India. London: British Library Publications.

HASAN Shah 1992 The Nautch Girl. Engl. trs. by Qurratulain Hyder. New Delhi:Sterling Publishers.

KERSENBOOM Saskia C. 1987 Nityasumangalī, Devadasi Tradition in South India. Delhi:Motilal

Banarsidass.

KIPPEN James 1988 The Tablā of Lucknow. Cambridge: Cambridge University Press.

ITC (Sangeet Research Academy Forum) 2004 Music Research: a Focus on Musical Forms. Mumbai: NCPA.

LAKSHMI C. S. 2000 The Singer and the Song. New Delhi:Kali for Women.

MACISZEWSKY Amelia 1998 Gendered Stories, Gendered Styles: Contemporary Hindusthani Music as Discourse, Attitude and Practice. Austin:University of Texas at Austin, PhD.

MANUEL Peter 1989 Thumri in Historical and Stylistic Perspective. New Delhi: Motilal Banarsidass.

MOTI Chandra 1973 The World of Courtesans. New Delhi: Vikas Publishing House.

NEVILE Pran 1996 Nautch Girls of India, Dancers, Singers, Playmates. New Delhi : Ravi Kumar Publisher.

POST Jennifer 1988 «Professional Women in Indian Music : the Death of the Courtesan Tradition ", in Women and Music in Cross-Cultural Perspective. Chicago : Chicago University Press : 97-108.

PUNJAB PURITY ASSOCIATION Opinions on the Nautch Question, collected and published by the Punjab Purity Association. Lahore: New Lyall Press.

PUKRAJ Malka 2003 Song Sung True, a Memoir. New Delhi: Kali for Women.

RAGHUNATHJI K. 1884 « Bombay Dancing Girls », The Indian Antiquary 13: 165-178. 
RUSWA Mirza Mohammad 1996 The Courtesan of Lucknow (Umrao Jan Ada). Engl. Tr. by David Matthews. Calcutta: Rupa \& Co.

SHARAR Abdul 2001 Hindusthan men Mashriqi Tamaddun ka Akhri Namunai ('The Last Example of Oriental Culture in India'), edited by E. S. Harcourt and Fakhir Hussain. New Delhi: Oxford University Press.

SHATTAN Merchant-Prince 1989 Maniimekhalai (The Dancer with the Magic Bowl). Engl. Tr. Alain Danielou. New York: New Directions.

The Christian Literature Society 1893 Nautch Women: an Appeal to English Ladies on Behalf of their Indian Sisters. Madras:S.P.C.K. Press.

VATSYAYANA 1994 Kamasutra, Eng. Tr. By Alain Danielou. Rochester : Inner Traditions India.

\section{NOTES}

1. L'adjectif hindoustani détermine la tradition culturelle de l'Inde du Nord. Il dérive de Hindusthan, nom donné par les envahisseurs musulmans à leurs conquêtes en Inde.

2. A l'exception de Mirabai, une poétesse musicienne du XVIe siècle, qui est encore aujourd'hui vénérée comme une sainte à cause de l'aspect dévotionnel de son art (Bahadu 1998).

3. Cet article s'inscrit dans le cadre d'une recherche entreprise entre 2000 et 2004 en Inde du Nord, et notamment à l'Université hindoue de Bénarès, pour le compte de l'Université la Sapienza de Rome.

4. C'est pourquoi je pense qu'il est nécessaire d'établir une classification - qui n'a fait à ce jour l'objet d'aucune étude - pour préciser les typologies et les dénominations artistiques correspondant à ces catégories. Ceci permettra d'éviter toute confusion entre des phénomènes qui sont séparés par des périodes et des cultures différentes, tout en favorisant l'identification de la catégorie des bāijī comme les héritières contemporaines d'une longue tradition de chanteuses et de danseuses de l'Inde du Nord.

5. Des informations intéressantes à ce sujet proviennent également de romans et d'autobiographies, Umrao Jahan Ada et The Natch Girl (eighteenth century), Malka Pukraj Memoirs (Pukraj 2003).

6. Le nath utāmā est également cité dans un passage de Natch girl, le premier roman de littérature urdu écrit par Hasan Shah en 1772. Dans la traduction anglaise de cette œuvre qui raconte l'histoire d'une tawā'if, le lettré Qurratulain Hyder explique qu'il y avait, en plus du nath utāmā, une autre cérémonie appelée missi, qui sanctionnait le nouveau statut de la fille : « Le jour où la fille vierge d'une courtisane était initiée à la profession, elle était parée comme une jeune mariée. Son premier protecteur ôtait son anneau de nez pour le remplacer par un bijou en forme de fleur. La cérémonie était célébrée comme un mariage auquel étaient conviés tous les membres de la caste de la courtisane pour festoyer pendant toute la nuit en dansant et en chantant. Les frais de la fête étaient assumés par l' 'initiateur', qui était la plupart du temps l'enchérisseur le plus riche de cette occasion convoitée. Le rite s'appelait missi, du nom d'une peinture noire que la jeune fille appliquait pour la première fois sur ses gencives. Il s'agissait d'une des seize décorations du maquillage traditionnel indien $»$.

7. En outre, les courtisanes qui n'avaient pas de filles adoptaient, achetaient et même kidnappaient des fillettes, pour ensuite les engager dans la profession, en leur transmettant à la fois un héritage artistique et de solides avantages matériels (Raghunathji 1884, Moti 1973).

8. En fait, ces termes servaient à désigner indifféremment les artistes des cours du Nord de l'Inde et les danseuses des temples du Sud du pays, les devadāsī, dont je ne n'occuperai pas ici (voir l'article d'Inoue ci-dessous, n.d.l.r.). 
9. A cause de l'intérêt suscité parmi les colons britanniques, le phénomène des filles nautch fut amplement documenté pendant la période de la domination anglaise, y compris par de nombreuses photographies, conservées aujourd'hui dans la section orientale de la British Library de Londres. Mieux qu'aucune autre description, ces documents photographiques montrent le vrai visage de ces femmes, dont le style varie d'une région à l'autre, et révèlent la grande disparité qui caractérisait la classe des courtisanes.

10. En Inde du Sud la tradition des devadāsī subit également le même sort. En 1947, on interdit officiellement à ces artistes de participer aux rituels du temple (Gaston 1996).

11. Entrevue avec Saira Begum à Bénarès, le 20 janvier 2003.

12. Le mujra est une réunion au cours de laquelle la tawā'if divertit un nombre restreint d'hommes.

13. Entrevue avec Saira Begum à Bénarès, le 20 janvier 2003.

14. «Les salons des courtisanes de luxe étaient des centres importants d'étiquette sociale aussi bien que de danse et de musique. Parfois des jeunes garçons de bonne famille y étaient envoyés à plusieurs reprises, accompagnés d'un adulte, pour parfaire leur éducation sociale. Ce genre de courtisanes dépendaient généralement d'un riche aristocrate, touchaient un salaire régulier et ne s'adonnaient pas à la prostitution. » (Sharar $2001: 276$ )

15. Apparemment seules quelques douzaines de kothās restent encore actifs, mais de façon presque clandestine à cause des risques de répression.

16. Le tablā est formé d'une paire de tambours accordés à l'unisson, l'un en métal (bāyān) dont la peau émet des sons graves, et l'autre en bois (dhāhinā) dont la peau émet des sons aigus.

17. Le sārangī est une vièle, c'est-à-dire un instrument à cordes frottées par un archet, avec une caisse de résonance en bois et un table en parchemin. Il est doté d'un manche large et court ne comportant pas de frettes, de trois cordes en boyaux et d'un jeu supplémentaire de cordes sympathiques.

18. Après plusieurs mois de recherche en Inde, je choisis de me concentrer sur la ville de Bénarès, jadis un important centre musical où vivent encore des bāijì.

19. Le rāga est la principale forme de la musique classique indienne.

20. Etant donné l'absence de familiarité avec le répertoire bāijī et la rareté des occasions offertes pour assister à leurs performances, toutes les études publiées jusqu'ici sur la 醚humrī se basent sur une version moderne affadie de ce genre musical interprété par les chanteurs classiques.

21. Le dhrupad et le khyāl sont les principaux genres de la musique de l'Inde du Nord. Le dhrupad qui se développa entre le $\mathrm{XVI}^{\mathrm{e}}$ et le XVIII ${ }^{\mathrm{e}}$ siècles, était rattaché à la tradition du temple ; il dégage une atmosphère majestueuse et sacrée. Le khyāl quant à lui, se développa entre le XVIII ${ }^{\mathrm{e}}$ et le $\mathrm{XIX}^{\mathrm{e}}$ siècles dans les cours princières ; il a un caractère plus romantique.

22. Entretien avec Saira Begum, 8 janvier 2003.

23. Pratique de la musique.

24. Entretien avec Saraswati Devi, Bénarès, $1^{\mathrm{er}}$ décembre 2002.

25. Extrait d'un entretien avec Ustad Rahim Fahimuddin Dagar, New Delhi, 23 mars 2004.

26. Entretien avec Ustad Rahim Fahimuddin Dagar, New Delhi, 27 juillet 2003. 


\section{RÉSUMÉS}

L'histoire de la musique indienne est connue à travers l'interpétation masculine de la tradition savante, laissant dans l'oubli la contribution artistique des musiciennes de cour, danseuses et chanteuses. En commentant certaines des motivations culturelles qui ont occulté l'activité des musiciennes courtisanes (bāijīi) en Inde, Francesca Cassio présente une brève analyse historique et artistique de la tradition féminine à partir de certaines descriptions figurant dans le Kāma Sutra, l'œuvre de Vatsyayana datant du IV ${ }^{\mathrm{e}}$ siècle de notre ère. L'auteure pense que, malgré les différences linguistiques et culturelles qui caractérisent les diverses classes d'artistes à des périodes et dans des environnements différents, on peut, en fait, concevoir un archétype qui, depuis l'époque de Vatsyayana jusqu'à nos jours, présente les mêmes caractéristiques sociales et artistiques. Pendant quatre années de recherche sur le terrain, Cassio a étudié l'activité d'une bāijī contemporaine, en analysant son répertoire de danse et de chant. La principale caractéristique de l'art des courtisanes était, en fait, la fusion de la musique et de la danse. Enfin, l'auteure fait une comparaison entre la gestuelle masculine employée dans la pratique de la musique classique et la gestuelle féminine utilisée dans le répertoire interprété par les bāijī, symbolisant une conception différente de l'esthétique musicale.

\section{AUTEURS}

\section{FRANCESCA CASSIO}

Francesca Cassio est chanteuse et ethnomusicologue, spécialisée dans la musique vocale hindoustanie. Docteur de l'Université de Rome, en association avec l'Université hindoue de Bénarès, elle fait des recherches de terrain en Inde depuis 1995. Elle enseigne l'ethnomusicologie indienne et le chant dhrupad au Conservatoire de Vicenza. Depuis 1995, elle étudie le dhrupad sous la direction de Ustad Rahim Fahimuddin Dagar ; plus récemment elle a entrepris de se

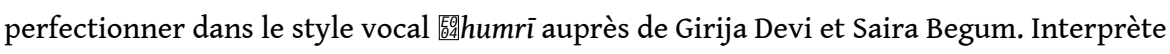
reconnue, Cassio a à son actif de nombreux enregistrements pour le théâtre, la radio, la télévision et le cinéma. 